\title{
La Torreta de Castielfabib, ¿una torre defensiva hexagonal tardomedieval? Álvaro Vázquez-Esparza
}

Universitat Politècnica de València, Valencia, España, alvazes@gmail.com

\begin{abstract}
Medieval fortifications placed in the inner part of the Valencian Country or even around its borders, are usually designed with simple shapes such as squares, rectangles or circles. Polygonal shapes appeared as well when it comes to the main towers of some fortresses like Puertomingalvo or Zafra, both close to the Aragon frontiers. The remains of the tower called "la Torreta" in Castielfabib (Valencia) remind us the pentagonal buildings quoted before, but according to the engraving made by Manfredo Fanti in 1840 this tower had an hexagonal design. Since no archaeological intervention has been done yet to clarify this issue, factors like: the lack of coherence between the present remains of the building and the only graphical information we have, the rarity of hexagonal shapes during the XIV-XV and the scarce of space to place certain geometrical figures, should be studied to move forward on this field.
\end{abstract}

Keywords: torre hexagonal, Castielfabib, Torreta, fortificación

\section{Introducción}

Frecuentemente un paño de muralla aislado, por sí solo, no nos desvela la historia o relevancia que alberga o las vicisitudes que ha sufrido hasta llegar a nuestros días, a diferencia del resto de las estructuras fortificadas que han sucumbido con el paso de los siglos. La Torreta de Castielfabib (comarca del Rincón, País Valencià) es un claro ejemplo de ello, asentada sobre el extremo oeste de la antigua Villa fortificada, un muro en ángulo se emerge sobre la parte más alta de la colina. Este elemento, a priori no parece ser más que un fragmento de la antigua muralla que protegía la población frente al enemigo castellano, pero es al conocer un grabado del s. XIX cuando nos apercibimos de que se trataba de una torre de gran empaque ubicada en la parte más vulnerable de todo el recinto y fuertemente fortificada.

No obstante, la geometría exacta de esta torre se desconoce ya que según el grabado descrito, se trata de un hexágono regular, ahora bien, esta geometría no es nada habitual en estas latitudes ni en el periodo histórico en el que se ubica la construcción de este edificio. Es por lo tanto objeto de esta investigación analizar en función de los datos documentales conocidos, la geometría todavía presente en los restos de la torre y casos similares conocidos al respecto, si la Torreta de Castielfabib tuvo la forma que los dibujos de 1840 muestran o no.

\section{Contexto histórico y geográfico}

Castielfabib es uno de los recintos fortificados más singulares que se conservan a lo largo de la ruta fluvial dentro del dominio valenciano que Lopez Elum (2002: 100) denomina como la del "Valle del Turia. Vía Valencia-Castielfabib",y que constituye el principal corredor de acceso desde el sur de Aragón hacia la capital del Reino de Valencia, a la vez que una línea defensiva fronteriza frente a los límites del Reino de Castilla en ese momento jalonada por multitud 
de castillos especialmente activos en el s.XIV, durante las sucesivas guerras con Castilla.

Del mismo modo que Ademuz, Castielfabib es conquistada por Pedro II, padre de Jaume I, en el año 1210, siendo consideradas Villas de Realengo una vez se establece el Reino de Valencia según el fuero de 1261 (Guinot, 1995), formando parte del brazo real de les Corts Valencianes donde tendrán voz y voto. Es, no obstante, su ubicación en la parte más alta del curso del Turia y la cercanía a la frontera castellana lo que hizo que fuesen muchos y abundantes los episodios bélicos tras la conquista cristiana ocasionando continuas modificaciones, destrozos y adaptaciones en la Villa (Almerich, 2011). Esta gran actividad durante la Guerra de los Pedros y otros conflictos de gran envergadura durante los siglos XIV-XV será lo que configurará la estructura defensiva que todavía hoy en día se distingue, y lo que justificará el alcance que durante finales de la Edad Media tuvo esta Villa. En cualquier caso, la decadencia iniciada a partir de finales del siglo XV con la unión de las Coronas de Aragón y Castilla hará que poco a poco las estructuras militares caigan en desuso, sobre todo el recinto urbano amurallado, y vayan siendo absorbidas por el desarrollo de la población. Un desarrollo que a lo largo de los siglos ha ido enmascarando, sustituyendo $\mathrm{y}$ reciclando las antiguas murallas como ha ocurrido en muchos otros lugares, si bien es cierto que en el caso de Castielfabib la pobreza, el aislamiento, la lejanía de grandes urbes y la consiguiente emigración ha permitido mantener en cierta medida gran parte de estos elementos. Solamente el Castillo-celoquia y la Torreta fueron utilizados $\mathrm{y}$ en parte reedificados de nuevo en el año 1839, durante la Primera Guerra Carlista por parte del bando carlista, siendo dinamitados por las tropas liberales de Isabel II una poco antes de finalizar la guerra en 1840. En cualquier caso, tras todas estas situaciones y desconociendo realmente el alcance de que tuvo todo este complejo, son muchas las estructuras defensivas que han llegado hasta nuestros días ya sea completamente en pie como la Iglesia-Fortaleza, semiarruinadas como el Castillo-celoquia y la denominada Torreta, o bien dispersos entre el tejido urbano varios lienzos de muralla y torres emergentes entre las viviendas.

\section{La torreta}

\subsection{Datos de partida y descripción}

De lo que se conoce como la Torreta, los restos llegados a nuestros días son escasos, solamente se conservan aquellas partes que resistieron la voladura de 1840, el paso del tiempo y el posterior expolio a modo de cantera. Concretamente, el muro sur de la torre con su cara interior lisa y exterior en ángulo, y partes de dos aterrazamientos sobre la ladera, además de gran cantidad de escombros que impide apreciar con exactitud la geometría de ésta pieza.

El muro sur, la parte más visible de todo el conjunto, tiene unas dimensiones de 6 metros de ancho y $10 \mathrm{~m}$ de altura desde la parte más baja del talud inferior. Constructivamente presenta un talud inclinado de piedra oscura creado para tener una plataforma estable donde asentar la torre. Por lo que respecta al resto del paño, encontramos una hoja exterior construida con sillarejo de piedra caliza blanca a excepción de las esquinas en ángulo donde se emplea tosca, un material abundante en la zona y que debido a su fácil trabajabilidad es frecuente encontrar en forma de sillería en aquellas construcciones relevantes de la población. Por el interior la torre encontramos un encofrado con ripios $\mathrm{y}$ mampuestos en masa a modo de hormigón ciclópeo de cal, presente también en otras torres del conjunto, desconociendo si por este lado existió algún tipo de hoja de mampostería expoliada posteriormente o no; se aprecian además las tongadas irregulares de vertido de material. Además del ángulo de $135^{\circ}$ que se aprecia en el muro anteriormente descrito, en la cota de cimentación del lado oeste se distingue el ángulo con el paño contiguo, siendo éste ligeramente superior al otro existente con $140^{\circ}$ según la toma de datos realizada. En el lado este, en cambio, se puede percibir una estructura previa construida con mampostería y recubierta con yeso, de la cual se desconoce si se trata de una torre primigenia más sencilla demolida para 
levantar la Torreta, o bien parte de una torre original embebida dentro de la última fortificación de la cual se reaprovecha alguna parte.(Fig.1).
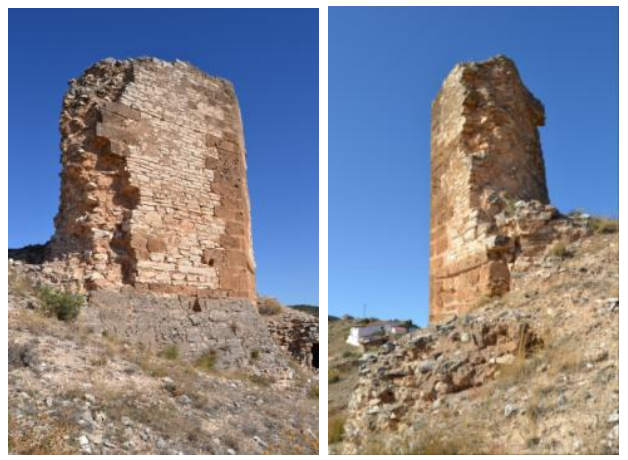

Fig.1-Vista oeste y este de la Torreta.

Los restos de las plataformas horizontales, presentan una construcción menos cuidada en general, distinguiendo en la parte ubicada más al sur los restos de un aparejo sillarejo de caliza y sillería basta de tosca relleno en gran parte por ripios o mampuestos para la creación de estancias reutilizadas como cortes de animales. En la vertiente este, se aprecia un aparejo de mampuestos con mucho mortero de mala factura comparado con el resto de las estructuras.

\subsection{Documentación existente}

No existe documentación explícita sobre la denominada Torreta como entidad diferenciada del castillo de Castielfabib, todas las referencias históricas que se han hallado hacen referencia al castillo en general. Incluso en aquellos casos en los que se especifican ciertas torres o elementos de todo el conjunto, la vaguedad de las referencias como "torre de acceso" cuando existen varios accesos, o la pérdida de la toponimia como es el caso de "la Torre de la Fe", donde no existe correspondencia con ninguna de las actuales, hace que los posibles datos de libros de obra, descripciones, etc. sean de difícil utilidad por lo menos con el grado de conocimiento actual del caso de Castielfabib. No obstante son frecuentes las órdenes de obras genéricas desde finales del s. XIII y ya en el s.XIV se hallan expedientes de obra detallados y a medida que avanza la centuria las reformas se vuelven más frecuentes fruto de los conflictos con el vecino reino de Castilla. Durante el siglo $\mathrm{XV}$ también existen múltiples intervenciones en la celoquia del castillo, y ya es en el siglo XVI cuando comienza el declive de la fortaleza en su conjunto hasta el siglo XIX (Eslava,2014).

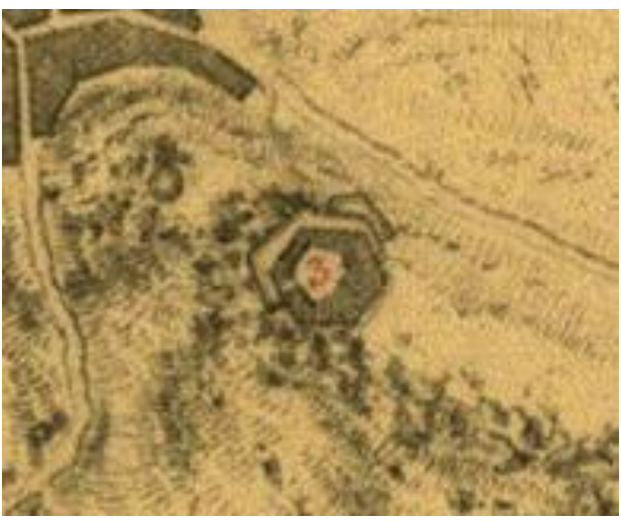

Fig.2- Planta de la torreta en el grabado de M. Fanti, 1840. Centro Geográfico del Ejército.

Es durante el desarrollo de la Primera Guerra Carlista (1833-1840), cuando en el año 1839 las tropas carlistas empiezan a fortificar el antiguo castillo medieval de Castielfabib, reaprovechando partes y construyendo otras nuevas para hacer frente a las necesidades del momento. Procede de este periodo la documentación gráfica más relevante conservada hasta la fecha, ubicada en el Centro Geográfico del Ejército, y se trata de un grabado levantado por el general constitucional Manfredo Fanti a principios de 1840 previo a la demolición del sistema defensivo de la Villa, incluida la Torreta. Este grabado titulado "Reconocimiento hecho por las tropas constitucionales sobre los fuertes enemigos, Castiel o Castielfavit, en el dia 6 de enero de 1840", recoge diversas imágenes como son un mapa topográfico de la población donde se distingue la supuesta planta hexagonal de la Torreta (Fig.2), aunque lo más relevante son las secciones detalladas de ésta y la celoquia, acotadas en varas castellanas (Fig.3), así como la perspectiva un tanto forzada tomada desde el suroeste donde se puede apreciar todo el conjunto en este periodo concreto (Fig.4). En los detalles grafiados en este documento se distingue en un tono rojo los restos del castillo 
existentes y en granate las nuevas estructuras levantadas por el bando Carlista, no obstante al tratarse de acuarela y tonos similares, en ocasiones no resulta fácil distinguir un tono del otro en la actualidad.

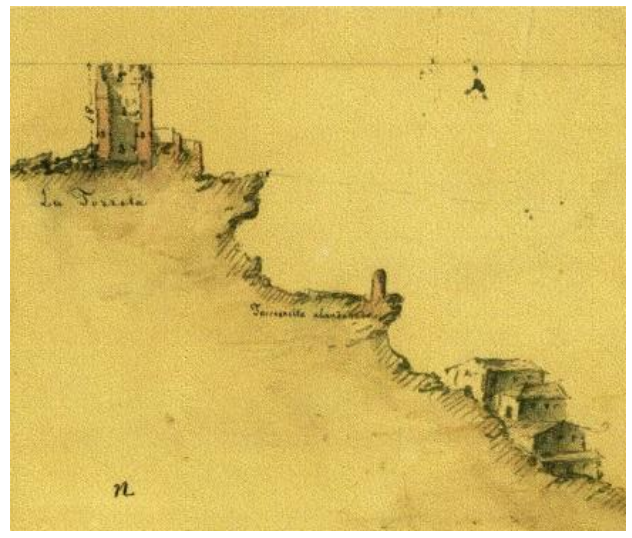

Fig.3- Sección de la Torreta según el grabado de M. Fanti, 1840. Centro Geográfico del Ejército.

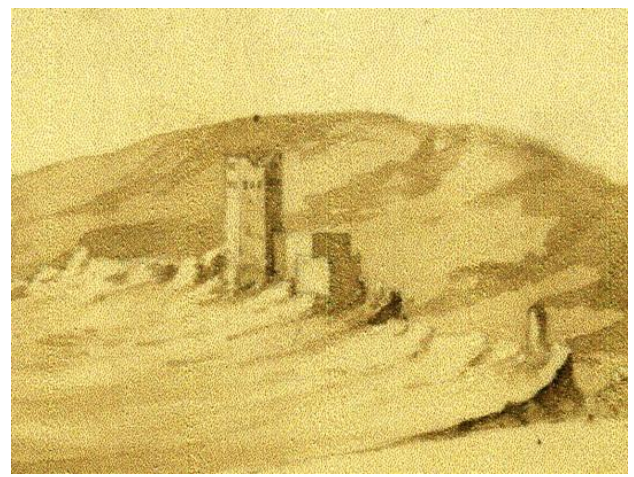

Fig.4- Perspectiva de la torreta en el grabado de M. Fanti, 1840. Centro Geográfico del Ejército.

El detalle con el que esta levantado el grabado anteriormente descrito presenta también algunas incongruencias, así como falsas perspectivas muy forzadas que pueden dar pie a malas interpretaciones o a hipótesis en ocasiones fuera de lugar. En cualquier caso, si se estudia la parte en la que se dibuja la Torreta, destaca sobre todo el gran tamaño de esta pieza como una torre albarrana probablemente articulada con el resto del recinto amurallado, pero que en este periodo, una vez ya desaparecidas las murallas de la población, aparece a modo de fortín independiente con dos plataformas exteriores y una altura total de 15 metros. Resulta llamativo que dada la envergadura de este elemento, su erección durante el siglo XIV según el arqueólogo Bruno Rives y el historiador Raúl Eslava, su emplazamiento en el punto más crítico de las defensas de Castielfabib durante las guerras con Castilla y el control de los accesos por el oeste no aparezca citado de forma explícita o al menos no se haya sabido reconocer en la documentación existente.

Hay quien ha sugerido que este edificio pudo levantarse en siglos posteriores o incluso en periodo carlista, aunque lai elaborada factura de la torre, su relevante protección por el oeste y el poco tiempo que tuvo el bando carlista para fortificar Castielfabib, así como la mala factura de las intervenciones carlistas halladas en otros lugares remiten al origen tardomedieval de los actuales restos.

Atendiendo a la descripción en sección de la Torreta, vemos que la altura total es de 18 varas castellanas, y considerando que la vara castellana utilizada mide $0,8359 \mathrm{~m}$, resultan unos $15 \mathrm{~m}$ de altura total, subdividida en 3 partes: planta baja, planta primera con saeteras o similar y un último nivel donde se hallan las almenas a modo de cubierta. Cada uno de estos niveles tiene unas proporciones diferentes en cuanto a anchura y presumiblemente altura, aunque únicamente se especifica los anchos y gruesos de muros. De este modo la estancia del nivel más bajo mediría 3 varas de ancho y 3 varas cada uno de los muros que lo delimitan, en metros 3 varas serían $2,47 \mathrm{~m}$.

En la planta primera en cambio el tamaño de la estancia aumenta a 4 varas $(3,34 \mathrm{~m})$ y en consecuencia los muros exteriores se reducen a 2,5 varas $(2 \mathrm{~m})$. Ya en el último nivel, los muros exteriores almenados miden 1,5 varas $(1,25 \mathrm{~m}) \mathrm{y}$ el espacio interior 6 varas $(5 \mathrm{~m})$. Tomando como referencia el grabado, se ha dibujado de nuevo la sección de la torre con las medidas descritas a escala con el objetivo de apreciar el grado de exactitud del documento (Fig.5) y de este modo conocer las proporciones reales de la torre. 

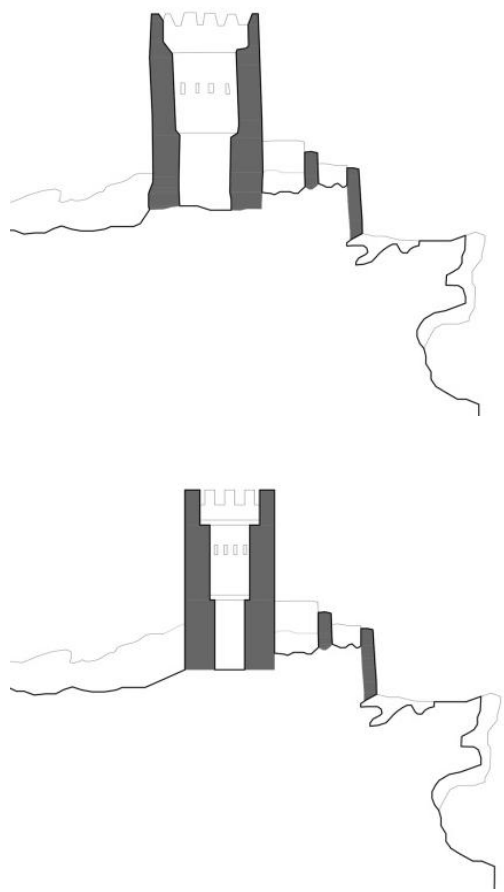

Fig.5- Arriba dibujo tomado directamente del grabado de 1840 , debajo la torre dibujada con las medidas indicadas en dicho documento.

\section{Casos similares}

Según el grabado de Fanti de 1840, la Torreta es un edificio hexagonal de planta relativamente regular, ahora bien no es nada frecuente la existencia de torres con esta geometría y de forma aislada en la zona en la que nos encontramos. En el caso de Castielfabib las torres que se mantienen del recinto amurallado presentan una planta cuadrada o rectangular más o menos regular, no obstante se trata de piezas secundarias dentro de todo el recinto. Sin embargo, sí que existen torres del homenaje o muy relevantes con plantas poligonales relativamente cerca tanto geográfica como históricamente, ahora bien, la mayoría de ellas presentan plantas pentagonales en proa (MoraFigueroa, 2006), formado por un pentágono irregular con dos de sus ángulos contiguos rectos $\left(90^{\circ}\right)$, asemejable también a una figura compuesta por un rectángulo y un triángulo en uno de sus lados para defenderse mejor del enemigo (Fig.6). Éste es el caso de Puertomingalvo (Teruel) (Fig.7), una fortaleza del siglo XIII que en algunos casos ha sido citada como posible símil al caso de la Torreta de Castielfabib. O el caso más lejano del castillo de Zafra, una fortificación ya en la parte de Castilla (provincia de Guadalajara) pero junto a la frontera aragonesa, donde una de las torres tiene una planta pentagonal irregular en uno de sus extremos. Otros casos son también la Torre de Aragón, en Molina de Aragón, o una de las torres albarranas del castillo de Alarcón, por citar algunos ejemplos reconocibles. Es decir que no encontramos referencias sobre torres defensivas hexagonales en el ámbito geográfico en el que nos encontramos en ningún periodo histórico, hay que desplazarse hasta otras latitudes tanto fuera del antiguo Reino de Valencia como dentro para encontrar algún caso similar, es el caso de las torres de la Porta dels Serrans en València, la porta de Sant Miquel de Morella o la Porta Reial del Monestir de Poblet, en este caso pareadas flanqueando los accesos pertinentes. Todas estas torres se construyen durante la segunda mitad del siglo XIV, no forman un hexágono regular y son elementos muy simbólicos construidos en el periodo de mayor agitación entre las coronas de Aragón y Castilla, del mismo modo que ocurre en el caso de Castielfabib.
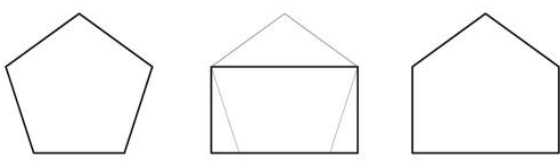

Fig.6- Esquema y evolución del trazado de torre pentagonal en proa.

De tal manera y poniendo en tela de juicio la geometría dibujada por el grabado de Manfredo Fanti en 1840, debido a la singularidad que supondría y teniendo como referencia otros casos similares, no es descabellado tal vez confiar en el dibujo referido, teniendo como referencia casos más lejanos geográficamente pero viables históricamente aunque ello suponga ajustar la versión que el documento de 1840 nos aporta. 


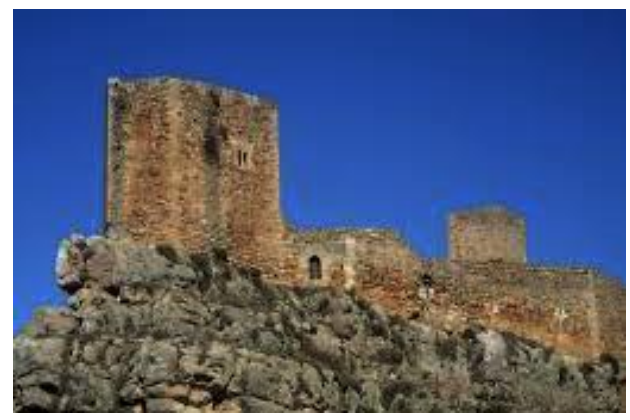

Fig.7- Torre pentagonal del Castillo de Puertomingalvo (Teruel).

\section{Hipótesis}

Llegado a este punto, una vez analizados todos los datos disponibles sobre este caso, así como sondeadas las diferentes opciones que se barajan, podemos establecer que la actual torre con toda probabilidad sustituye a otra más humilde ubicada en el mismo lugar debido a su posición estratégica, y a los restos que se pueden apreciar in situ a falta de una excavación arqueológica. Incluso se puede interpretar que la presente torre se materializa como refuerzo, por su parte sur por lo menos, de la torre anterior dotándola de la forma geométrica posterior. Ahora bien, los restos de la torre actual, ¿con qué esquema en planta se relacionan?

- La referencia a una torre hexagonal se manifiesta únicamente en el grabado de 1840, el cual aun siendo un documento muy detallado como se ha especificado anteriormente, manifiesta fallos o faltas de precisión que podrían justificar el dibujo de una torre con una geometría en planta diferente a la real. Planteando una torre con un trazado en planta irregular del cual con los datos existentes hasta la fecha no se podría afirmar el número de lados exacto (Fig.8).

- A pesar de que existen diversas torres pentagonales en proa en situaciones muy similares a la Torreta de Castielfabib, como las citadas anteriormente, los dos ángulos distinguibles de 135 y $140^{\circ}$ no se corresponden con esta geometría. Si se hubiese tratado de un único ángulo de entorno a $135^{\circ}$, podría haber sido indicador de planta pentagonal en proa, pero con dos ángulos de este calibre la opción del pentágono en proa debe descartarse, si bien no la de pentágono irregular que podría ser viable.

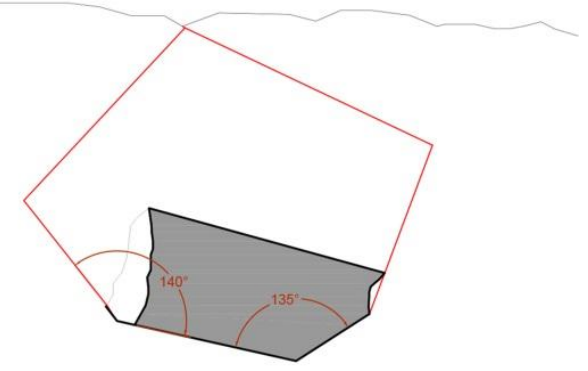

Fig.8- Hipótesis de polígono irregular manteniendo los dos ángulos existentes.

- La opción de un hexágono regular, tal y como muestra la documentación gráfica no parece evidente, de tal modo que es razonable pensar en un hexágono pero irregular o "en proa", siguiendo el esquema de la torre pentagonal en proa, es decir un hexágono irregular con dos ángulos contiguos rectos (Fig.9), como muestran los casos de las Torres dels Serrans (1392-1398) obra de Pere Balaguer y precedidas por las torres de la Porta de Sant Miquel de Morella (1358-1362) de Domènec Travall o la Porta Reial del Monestir de Poblet (1367-1382), todas ellas de sillería y rematadas por matacanes y aspilleras (Cervera et al, 2003). Salvando las distancias en cuanto a relevancia o composición de cada uno de los casos, la hipótesis de una estructura en planta extrapolable de los citados casos a la Torreta, sería totalmente viable. Además, los ángulos hallados de $135^{\circ}$ cuadrarían con esta tipología de torre.

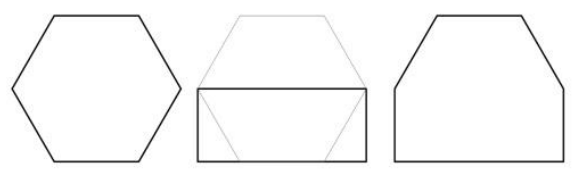

Fig.9- Esquema y evolución del trazado de torre hexagonal "en proa".

- Existen otra opción donde la torre que nos ocupa tuviese una geometría diferente a los casos anteriormente planteados si se interpreta 
de un modo estricto el ángulo del lado sur. Este ángulo de $135^{\circ}$ es el que forman las aristas de un octógono regular, ahora bien la geometría que describiría este tipo de trazado no cabría en el espacio existente en las inmediaciones de la torreta según los datos manejados (Fig.10).

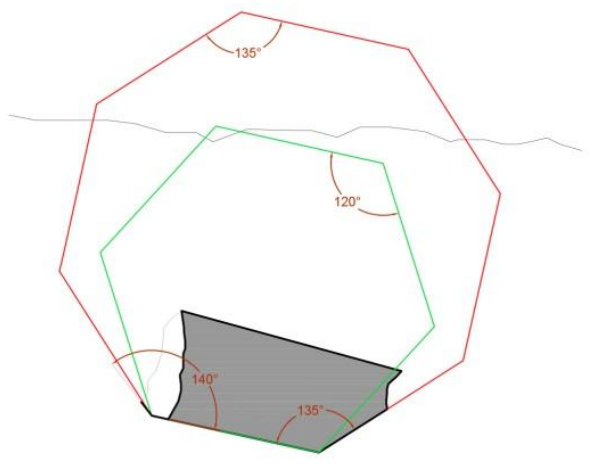

Fig.10- Hipótesis de hexágono u octógono regular.

\section{Conclusiones}

Solamente una excavación arqueológica que defina de un modo real las características formales de la Torreta, o bien nueva documentación que arroje luz a la presente investigación nos permitirá conocer a ciencia cierta cuál es la geometría real de este caso. Lo que sí que parece claro es la existencia de otra torre previa, la cual es sustituida o reaprovechada en parte para la erección del nuevo edificio. No obstante una vez analizados todos los datos conocidos tanto a nivel empírico como documental podemos concluir que las hipótesis de que el trazado en planta de la Torreta sea un pentágono de planta irregular o bien un hexágono "en proa", que estaría formado por un hexágono irregular con dos ángulos contiguos rectos como se ha descrito anteriormente. Además, los ángulos existentes corroboran esta última hipótesis, puesto que si el trapecio de la figura fuese de $45^{\circ}$, sumado a los $90^{\circ}$ del ángulo recto del rectángulo obtendríamos un ángulo de $135^{\circ}$ que es el que existe en la fachada sur de la torre de estudio, asumiendo que el otro ángulo de $140^{\circ}$ puede deberse a una pequeña desviación o incluso a una deficiente toma de datos ya que solamente se conservan las dos piedras de arranque de esta esquina (Fig.11).

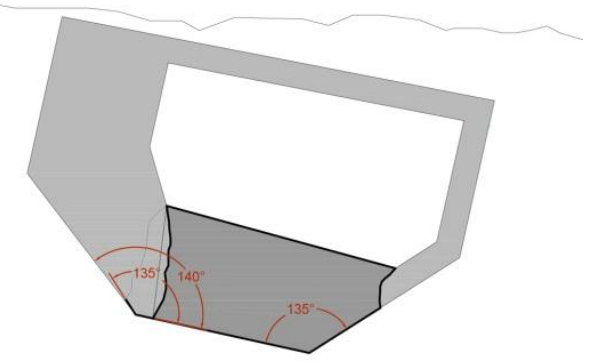

Fig.11- Propuesta de planta hexagonal "en proa" para la Torreta.

Validando por tanto esta teoría, se alcanzaría una solución que se corresponde tanto con los datos gráficos del grabado de Manferdo Fanti de 1840, así como con la información que arrojan las propias ruinas del edificio. Sin embargo asumir esta tipología de torre es también renunciar a la que parece ser más propia del lugar, en función de los casos existentes en su entorno geográfico e histórico, y por lo tanto la consiguiente novedad de este tipo de geometría en las latitudes y periodo donde nos encontramos. Ahora bien, quedaría pendiente verificar la eficacia de este tipo de arquitectura defensiva en un contexto bélico, puesto que no disponemos de referencias al respecto debido a la escasez de esta morfología.

\section{Referencias}

Almerich, J.M., (2011) Paisatges fortificats. Torres, muralles y castells a les terres valencianes.

$1^{a}$ Edición. Alzira, Editorial Bromera.

Cervera Arias, F., y Mileto, C., (2003) Las Torres de Serranos. Historia y restauración. Valencia.

Ajuntament de València.

Eslava Blasco, R., (2014) Castielfabib y su patrimonio histórico artístico. Castielfabib (Valencia). Ayuntamiento de Castielfabib.

Guinot E., (1995) Els límits del Regne.El procés de formació territorial del País Valencià medieval 
(1238-1500). Col•lecció Politècnica 58. València. Edicions Alfons el Magnànim-Institució valenciana d'estudis i investigación/Generalitat Valenciana-Diputació de València.

López Elum, P., (2002) Los Castillos valencianos en la Edad Media. Vol. II, Valencia, Biblioteca Valenciana.

Mora-Figueroa, L., (2006) Glosario de Arquitectura Defensiva Medieval. $3^{\text {a }}$ edición. Madrid. Ministerio de Defensa. 